Communications in Physics, Vol. 24, No.3S2 (2014), pp. 13-17

DOI:10.15625/0868-3166/24/3S2/5016

\title{
THE 3-3-1 MODELS IN CURRENT PARTICLE PHYSICS
}

\author{
PHUNG VAN DONG, LE THO HUE, DO THI HUONG, HOANG NGOC LONG \\ Institute of Physics, Vietnam Academy of Science and Technology \\ E-mail:pvdong@iop.vast.ac.vn
}

Received 20 June 2014

Accepted for publication 20 August 2014

\begin{abstract}
We review the main contribution of the Division for Theoretical Particle Physics at the Institute of Physics, $V A S T$ in building the models based on $S U(3)_{C} \otimes S U(3)_{L} \otimes U(1)_{X}$ gauge unification.
\end{abstract}

Keywords: unified theories and models of strong and electroweak interactions, extensions of electroweak gauge sector.

\section{INTRODUCTION}

After the discovery of the Higgs boson at the LHC by ATLAS and CMS [1], the last missing particle of the Standard Model (SM) has been found. The SM that provides a quite precise description of particle physics up to the electro-weak (EW) scale is one of the greatest successes of physics in the twentieth century. However, the neutrino mass and mixing are the first evidence of the beyond Standard Model. With the LHC discovery, the Higgs physics is an attractive subject. It is essential for us to determine which model the discovered Higgs boson belongs to? For this aim, the diphoton decay of the Higgs boson plays very important role. It is expected that new physics might enter here to modify the SM Higgs property. Moreover, the SM explains only about $5 \%$ mass-energy density of our universe. There remains around $25 \%$ dark matter and around $70 \%$ dark energy that the SM leaves unsolved. The SM cannot address the baryon asymmetry of the observable universe too.

We will give a brief review of the 3-3-1 models since they are our contributions to the development of particle physics at the Hanoi Institute of Physics as providing a potential solution for the most questions mentioned above.

\section{THE 3-3-1 MODELS}

Among the possible extensions of SM, a curious choice are the 3-3-1 models which encompass a class of models based on the gauge group $\mathrm{SU}(3)_{C} \otimes \mathrm{SU}(3)_{L} \otimes \mathrm{U}(1)_{X}[2,3]$, that is at first spontaneously broken to the $\mathrm{SM}$ group $\mathrm{SU}(3)_{C} \otimes \mathrm{SU}(2)_{L} \otimes \mathrm{U}(1)_{Y}$ and then undergoes the spontaneously broken to $\mathrm{SU}(3)_{C} \otimes \mathrm{U}(1)_{Q}$. The extension of the gauge group with respect to SM leads to interesting consequences. The first one is that the requirement of anomaly cancellation together with that of asymptotic freedom of QCD implies that the number of generations must necessarily 
be equal to the number of colors, hence giving an explanation for the existence of three generations. Furthermore, quark generations should transform differently under the action of $S U(3)_{L}$. In particular, two quark generations should transform as triplets, one as an antitriplet. Choosing the latter to be the third generation, this different treatment could be at the origin of the large top mass.

A fundamental relation holds among some of the generators of the group:

$$
Q=T_{3}+\beta T_{8}+X
$$

where $Q$ indicates the electric charge, $T_{3}$ and $T_{8}$ are two of the $S U(3)$ generators and $X$ is the generator of $U(1)_{X} . \beta$ is a key parameter that defines a specific variant of the model.

\section{II.1. Typical 3-3-1 models}

There are two main versions of the 3-3-1 models as far as the lepton sectors are concerned. In the minimal version $\left(\beta=\frac{1}{\sqrt{3}}\right)$, the charge conjugation of the right-handed charged lepton for each generation is combined with the corresponding $S U(2)_{L}$ doublet of the left-handed leptons to form an $S U(3)$ triplet $\left(v, l, l^{c}\right)_{L}$. No extra leptons are needed and therefore it was called minimal 3-3-1 model [2]. Another version $\left(\beta=-\frac{1}{\sqrt{3}}\right)$ adds a left-handed anti-neutrino to each usual $S U(2)_{L}$ doublet of the left-handed leptons to form a triplet, i. e., $\left(v, l, v^{c}\right)_{L}$ [3]. These left-handed anti-neutrinos serve the role of the charge conjugation of the usual right-handed neutrinos which are required in the usual see-saw mechanism. Therefore such model was called as the 3-3-1 model with right-handed neutrinos (331RHN) [3].

It was shown that the minimal version is perturbatively reliable at the scale around $\Lambda=4-5$ $\mathrm{TeV}$ [4]. In addition, the minimal model requires quite large Higgs sector: three triplets and one sextet. Here we focus on the model with right-handed neutrinos, since the resulting scenario turns out to be phenomenologically more interesting than other variants. Moreover, the new gauge bosons, that are present due to the enlarged gauge group, have integer charges for this value of $\beta$.

The model comprises several new particles. There are new gauge bosons $Y$ and $V$, whose charges depend on the considered variant. In 331RHN they are a singly charged $Y^{ \pm}$boson and a neutral one $X^{0}\left(\bar{X}^{0}\right)$. In all the variants a new neutral gauge boson $Z^{\prime}$ is present. This represents a very appealing feature, since $Z^{\prime}$ mediates tree level flavor changing neutral currents (FCNC) [5] in the quark sector (couplings to leptons are instead universal). An extended Higgs sector is also present, with three $S U(3)_{L}$ triplets and one sextet. Finally, new heavy fermions are predicted.

\section{II.2. 3-3-1 models with flavor symmetries}

The 3-3-1 models can be combined with discrete flavour symmetry such as $A_{4}$ [6], $S_{4}$ [7], $S_{3}$ [8] and $D_{4}$ [9] to provide not only tribi-maximal neutrino mixing but also the current neutrino data with non-zero $\theta_{13}$. These flavor symmetry extensions are natural since the 3-3-1 models by themselves provide the 3-dimentional, 2-dementional representations of lepton and/or quark families. In addition, the seesaw mechanism for small neutrino masses is automatic in these models.

Two kinds of the 3-3-1 models which can work under the family symmetries yielding the realistic neutrino mass spectrum are the $331 \mathrm{RHN}$ and the 3-3-1 model with neutral fermions: The former is with $S_{3}$, while the latter naturally fit all the discrete groups given. 


\section{II.3. Simple 3-3-1 models}

One of the weaknesses of the mentioned 3-3-1 models that reduces their predictive possibility is a plenty or complication in the scalar sectors. The attempt on this direction to realize simpler scalar sectors has recently been constructed 3-3-1 model with minimal Higgs sector called the economical 3-3-1 model [10,11]. The 3-3-1 model with minimal content of fermions and Higgs sector (called the reduced minimal (RM) 3-3-1 model) has also been constructed in [12]. However, in order to give all fermions masses, the effective interactions have to be introduced.

In these models, the total lepton number is broken due to some VEV and/or effective mass operators. The economical 3-3-1 model predicts abnormal lepton-number violating currents beyond the SM and typical 3-3-1 models. The Peccei-Quinn symmetry can automatically be imposed, but it will completely broken when all the quarks get masses. There is no axion. In the reduced 3-3-1 model, the axion is absent too. However, the phenomenology of the reduced 3-3-1 model is distinguished from the economical 3-3-1 model.

\section{II.4. Supersymmetric 3-3-1 models}

It is well known that the supersymmetric models consist of intriguing features such as the Higgs self couplings are almost the gauge ones. Therefore if supersymmetry is realized in Nature, there are some scalar fields have the same masses of gauge bosons. The supersymmetric version of the economical model was constructed in [13], and for the reduced minimal in [14]. The Higgs sector in the supersymmetric economical 3-3-1 model was considered in [15]. The family lepton number violating decays of Higgs bosons in this model was presented in [17], for decay of $Z$ boson in [18].

Supersymmetric (SUSY) versions were constructed to solve some problems occurring in the original 3-3-1 models such as fermion masses and dark matter. Fermion masses now can be generated by loop corrections of SUSY particles. Furthermore, the appearance of effective interactions in 3-3-1 models can be explained by these loop corrections. The dark matter candidate exists naturally as lightest superpartner particle under the $R$-parity conversation. The Higgs sector in the supersymmetric economical 3-3-1 model was considered in [15]. In these versions, the $B / \mu$ terms were not included leading to the existing of many massless scalar bosons in the Higgs sector. This will cause many dangerous invisible decay modes. The Higgs revised sectors were studied in [16] including $B / \mu$-terms. It can be shown that in these SUSY 3-3-1 models, all Higgses are massive and soft parameters are in range of $S U(3)_{L}$ scale. But there always exits a lightest CP-neutral Higgs with mass being smaller than $m_{Z}$ at tree level. Of course, this Higgs mass needs rather large correction from loop contributions to gain consistent mass of Higgs discovered recently at LHC. In general, Higgs sector in these models are very rich because they contain rather light charged Higgses that can be detected by experiments. Some other phenomenological aspects have also been investigated. For example, the family lepton number violating decays of Higgs bosons was presented in [17], for decay of $Z$ boson and $\tau$ in [18]. These decays as signals of new physics beyond SM have been hunting by recent colliders.

\section{II.5. 3-3-1 models for dark matter}

There exist many incarnations of 3-3-1 models in the literature, and many of them actually do not offer any viable DM candidate: these include the reduced minimal 3-3-1, and the economical 3-3-1 model, among others. Dark matter is recently attractive subject in particle physics. Dong 
and collaborators [19] have constructed the modified version containing rules for finding the dark matter.

If the 3-3-1 model respects the conservation of lepton and baryon numbers, the 3-3-1 symmetry has to be extended to the 3-3-1-1 symmetry with an extra $U(1)_{N}$ group in order to keep it (our 3-3-1 model) self-consistent. The $N$-charge is broken down to a $Z_{2}$ symmetry due to the spontaneous gauge symmetry breaking, called $W$-parity, similarly to $R$-parity in supersymmetry. The lightest particle that carries an anomalous lepton number as in the 3-3-1 model with neutral fermion can be dark matter because it is $W$-odd.

In other realizations, the 3-3-1 model with right-handed neutrinos as well as the minimal 33-1 model can by themselves work as the simple 3-3-1 models under a $Z_{2}$ symmetry, respectively, while they provide also inert scalar triplets responsible for dark matter.

\section{II.6. 3-3-1 models and cosmology}

Cosmological aspects in the framework of the 3-3-1 models were considered in [20-22]. In [20], the cosmological inflation was considered in the supersymmetric economical 3-3-1 model. More precisely, we studied a hybrid inflationary scheme based on a realistic supersymmetric 3-3-1 model by adding a singlet superfield $\Phi$ which plays the role of the inflation, namely the inflaton superfield. By studying the impact of the spontaneous symmetry breaking pattern from choosing model down to the SM, it gives rise to a false vacuum hybrid inflationary scenario which solves few problems of cosmology. The authors have studied the prediction of inflation contribution to the Cosmic Microwave Background (CMB) for the two kinds of supersymmetric hybrid inflation. The authors have shown that they are both in agreement with CMB data. The strong limit on the allowed the cosmic string contribution can be translated to constraint on all parameters in the inflationary models, such as coupling constant, energy scales of inflation, etc. We would like to emphasize that the supersymmetric models with inflation scenario have the lepton-number violation which plays very important role in the formation of Early Universe such as lepto/ baryogenesis. These phenomenology was studied in [21]. On the other hand, in [22] the electroweak phase transition in the reduced minimal 3-3-1 model was presented. It is interesting to note that by considering the phase transition, the constraints on masses and couplings in the scalar sector were derived.

It is to be noted that all the 3-3-1 model consist of CP asymmetry and family lepton number violation [23], the direct consequence of which is the universe matter-antimatter asymmetry. The values of $M_{Z \text { ? }}$ considered in these papers are sufficiently low that this new gauge boson could be discovered in the next run of the LHC and its properties could even be studied at a future ILC.

\section{CONCLUSIONS}

Let us summary this review by remarks on experiments providing data for checking correctness of the 3-3-1 models

(1) The LHC gives data on new particles such as new gauge bosons with lepton number two (bilepton) and Higgs scalars different from that of the SM. Dark matter is searched at the LHC too.

(2) Neutrino experiments around all the world provide data on neutrino mass and mixing, which are interesting for the 3-3-1 models with flavor discrete symmetries. 
(3) Cosmological data from the WMAP and PLANCK gives information inside study on the Early Universe in the 3-3-1 models.

It should be emphasized that the recent important contributions to the 3-3-1 models belong to the Institute of Physics, VAST.

\section{ACKNOWLEDGMENTS}

This research is funded by Vietnam National Foundation for Science and Technology Development (NAFOSTED) under grant number 103.01-2014.51.

\section{REFERENCES}

[1] G. Aad et al. [The ATLAST Collaboration], Phys. Lett. B 716 (2012) 1; S. Chatrchyan et al. [The CMS Collaboration], Phys. Lett. B 716 (2012) 30.

[2] F. Pisano and V. Pleitez, Phys. Rev. D 46, (1992) 410; P. H. Frampton, Phys. Rev. Lett. 69 (1992) 2889; R. Foot et al, Phys. Rev. D 47 (1993) 4158.

[3] M. Singer, J. W. F. Valle, and J. Schechter, Phys. Rev. D 22 (1980) 738; R. Foot, H. N. Long, and Tuan A. Tran, Phys. Rev. D 50 (1994) R34; J. C. Montero, F. Pisano, and V. Pleitez, Phys. Rev. D 47 (1993) 2918; H. N. Long, Phys. Rev. D 53 (1996) 437; Phys. Rev. D 54 (1996) 4691.

[4] A. G. Dias, R. Martinez, V. Pleitez, Eur. Phys. J. C 39 (2005) 101. See also, A. G. Dias, V. Pleitez, Phys. Rev. D 80 (2009) 056007.

[5] J. T. Liu, Phys. Rev. D 50 (1994) 542; D. G. Dumm, F. Pisano, and V. Pleitez, Mod. Phys. Lett. A 9 (1994) 1609; T. H. Lee and D. S. Hwang, Int. J. Mod. Phys. A 12 (1997) 4411; H. N. Long and V. T. Van, J. Phys. G 25 (1999) 2319; A. C. B. Machado, J. C. Montero, and V. Pleitez, Phys. Rev. D 88 (2014) 113002.

[6] P. V. Dong, L. T. Hue, H. N. Long and D. V. Soa, Phys. Rev. D 81 (2010) 053004.

[7] P. V. Dong, H. N. Long, D. V. Soa, and V. V. Vien, Eur. Phys. J. C. 71 (2011) 1544.

[8] P. V. Dong, H. N. Long, C. H. Nam and V. V. Vien, Phys. Rev. D 85, (2012) 053001.

[9] V. V. Vien and H. N. Long, Int. J. Mod. Phys. A 28(32) (2013) 1350159.

[10] W. A. Ponce, Y. Giraldo and L. A. Sanchez, Phys. Rev. D 67 (2003) 075001.

[11] P. V. Dong, H. N. Long, D. T. Nhung and D. V. Soa, Phys. Rev. D 73 (2006) 035004; P. V. Dong, D. T. Huong, Tr. T. Huong and H. N. Long, Phys. Rev. D 74 (2006) 053003; P. V. Dong, H. T. Hung and H. N. Long, Phys. Rev. D 86 (2012) 033002, for a review, see: P. V. Dong and H. N. Long, Adv. High Energy Phys. 2008 (2008) 739492, [arXiv:0804.3239(hep-ph)].

[12] J. G. Ferreira, Jr, P. R. D. Pinheiro, C. A. de S. Pires and P. S. Rodrigues da Silva, Phys. Rev. D 84 (2011) 095019.

[13] P. V. Dong, D. T. Huong, M. C. Rodriguez and H. N. Long, Nucl. Phys. B 772 (2007) 150.

[14] D. T. Huong, L. T. Hue, M. C. Rodriguez and H. N. Long, Nucl. Phys. B 870, (2013) 293.

[15] P. V. Dong, D. T. Huong, N. T. Thuy and H. N. Long, Nucl. Phys. B 795 (2008) 361.

[16] D.T. Binh, L.T. Hue, D.T. Huong, H.N. Long, Eur. Phys. J. C 74(5) (2014) 2851; J. G. Ferreira, C. A. de S. Pires, P. S. Rodrigues da Silva, A. Sampieri, Phys. Rev. D 88 (2013) 105013.

[17] P. T. Giang, L. T. Hue, D. T. Huong and H. N. Long, Nucl. Phys. B 864 (2012) 85.

[18] L. T. Hue, D. T. Huong, and H. N. Long, Nucl. Phys. B 873 (2013) 207.

[19] P. V. Dong, T. D. Tham, H. T. Hung, Phys. Rev. D 87 (2013) 115003; P. V. Dong, T. Phong Nguyen, D. V. Soa, Phys. Rev. D 88 (2013) 095014.

[20] Do T. Huong and Hoang N. Long, Yad. Fiz. 73(5) (2010) 822; Phys. Atom. Nucl. 73 (2010).

[21] D. T. Huong, H. N. Long, J. Phys. G: Nucl. Part. Phys. 38 (2011) 015202.

[22] Vo Quoc Phong, Hoang Ngoc Long and Vo Thanh Van, Phys. Rev. D 88, (2013) 096009.

[23] A. J. Buras, F. De Fario and J. Girrbach, JHEP 1320 (2013) 116; A. J. Buras, F. De Fario and J. Girrbach, 331 models facing new $b \rightarrow s \mu^{+} \mu^{-}$data, arXiv:1311.6729 [hep-ph]. 